\title{
NÍVEL DE ATIVIDADE FÍSICA DE ESTUDANTES UNIVERSITÁRIOS COM E SEM DISTÚRBIO DA IMAGEM CORPORAL
}

\author{
Leandro Martinez Vargas \\ Faculdades Integradas de Itararé, Itararé, São Paulo, Brasil \\ José Roberto Herrera Cantorani \\ Faculdades Integradas de Itararé, Itararé, São Paulo, Brasil \\ Lilian Kelen Mauricio \\ Faculdades Integradas de Itararé, Itararé, São Paulo, Brasil \\ Silvielly Karoline Leonardo Annunciato \\ Faculdades Integradas de Itararé, Itararé, São Paulo, Brasil
}

\begin{abstract}
Resumo
O objetivo do presente estudo foi analisar a relação entre o Nível de Atividade Física (NAF) e a percepção da Imagem Corporal (IC) em estudantes de ensino superior. Participaram do estudo 339 acadêmicos de sete cursos de graduação, dos quais 166 eram homens e 173 eram mulheres. A avaliação do NAF foi realizada por meio do IPAQ, na sua versão longa. O instrumento utilizado para analisar a IC foi o Body Shape Questionnaire (BSQ). Os dados revelam alta incidência de níveis insuficientes de atividade física $(38,9 \%)$ e baixa prevalência de distorção da IC (12,6\%), independentemente do sexo e curso de graduação. Conclui-se que o sexo feminino apresentou maiores chances tanto para níveis insuficientes de prática de atividade física, quanto de manifestação de distúrbio da IC.
\end{abstract}

Palavras-chave: Atividade física; Imagem corporal; IMC; Estudantes universitários.

\section{Introdução}

Em estudos a respeito da IC, dois fatores são alvos de atenção: o papel da consciência corporal e os padrões sociais relacionados ao corpo e à beleza. A consciência corporal está relacionada ao processo de autonomia do corpo. Por meio dessa consciência, o indivíduo adquire conhecimento sobre o seu corpo e suas partes, e aprende a respeitá-lo e conhecer seus limites (MASTROIANNI et al., 2007). Mas os padrões sociais para o corpo induzem muitos indivíduos à busca, irrefletidamente, pela adequação a tais padrões (BARBOSA; MATOS; COSTA, 2011).

Essa preocupação com a IC no que diz respeito a uma situação de aceitabilidade social leva o indivíduo a uma condição de submissão a esses valores, e, muitas vezes, à perda da identidade pessoal (ROCHA, 2009).

Sobre tal contexto, Silva (2010) afirma que o processo de desvendar o corpo conduz o indivíduo a uma harmonia com o meio em que está inserido, contribuindo para que haja autonomia corporal e para que se efetive a libertação das imposições da sociedade.

$\mathrm{Na}$ área da Educação Física, grande parte do conhecimento científico reunido acerca do referido tema gira em torno da percepção subjetiva do indivíduo sobre a sua própria imagem física e sobre a sua relação com fatores ligados aos aspectos nutricionais, qualidade 
de vida, estilo de vida e prática de atividade física (CAMPANA; TAVARES, 2009; GONÇALVES, 2009).

A prática de atividade física influencia diretamente na alteração da IC, pois, tende a melhorar a satisfação corporal e a autoestima (GONÇALVES, 2009; WILLIAMS; CASH, 2001; VIEIRA et al., 2005). No entanto, para que essa satisfação seja plena, a atividade física deve objetivar não apenas a composição corporal, mas também a saúde (GONÇALVES, 2009).

Estudos relacionados à análise da IC em universitários mostram que esse público apresenta características peculiares que o torna vulnerável a mudanças drásticas no comportamento e hábitos de vida (COQUEIRO et al., 2008; QUIOCA et al., 2010; COSTA et al., 2010; MARTINS et al., 2012). Segundo Vieira et al. (2002), a aquisição de novos hábitos motivados pelo início da vida universitária - ficar mais tempo afastado da família, novas amizades e atividades de lazer noturnas, além das mudanças biológicas que ocorrem com a idade - fazem com que os jovens estejam mais vulneráveis a fatores que prejudicam a saúde.

É fato corrente, em estudos (COQUEIRO et al., 2008; MARTINS et al., 2012; QUIOCA et al., 2010; COSTA et al., 2010; JESEUS; AFONSO, 2011), que mais de 70\% dos universitários encontravam-se, de alguma forma, insatisfeitos com sua IC. Também é um fato comum em estudos dessa natureza, o desejo das mulheres em perder peso e dos homens em aumentar o peso (COQUEIRO et al., 2008; MARTINS et al., 2012; QUIOCA et al., 2010). Nesse contexto, Miranda et al. (2012) afirmam que independentemente da influência da cultura, as mulheres mostram ser mais insatisfeitas com o corpo do que os homens. Em estudo de Alvarenga et al. (2010), grande parte das mulheres com peso normal manifestaram-se insatisfeitas com sua IC, afirmando desejo de serem mais magras.

No Brasil, ainda são poucos os estudos que apresentam informações quanto à associação entre a insatisfação com a IC e a prática de atividade física em universitários (FERRARI; SILVA; PETROSKI, 2012). Bracht (2011), em pesquisa com essa população, identificou que a maioria dos indivíduos que praticavam níveis insuficientes de atividade física, ou eram sedentários, apresentavam algum nível de distorção e insatisfação com sua IC. No estudo de Hirota et al. (2012), foi levantado que as universitárias com alto NAF tendem a apresentar-se mais satisfeitas com sua IC. Em contrapartida, entre as com NAF baixo ou moderado percebeu-se algum nível de insatisfação.

Dessa forma, considerando que a prática de atividade física pode estar associada ao modo como as pessoas percebem a sua IC, e, considerando que o ingresso no ensino superior pode alterar o modo como o indivíduo se vê junto ao seu grupo social, o estudo tem como objetivo analisar a relação do NAF com a percepção da IC e fatores socioeconômicos e demográficos em estudantes de sete cursos de graduação.

\section{Metodologia}

A presente pesquisa, de característica quantitativa, descritiva e de levantamento, foi desenvolvida nas Faculdades Integradas de Itararé, localizada no município de Itararé - SP. A instituição oferece os cursos de graduação em Administração, Ciências Contábeis, Direito, Educação Física, História, Pedagogia e Sistemas de Informação.

A população foi composta por acadêmicos - ingressantes e concluintes, de ambos os sexos - matriculados nos cursos de graduação oferecidos pela instituição. Considerou-se como base populacional o total de 498 acadêmicos. A avaliação do NAF foi realizada por meio do IPAQ, na sua versão longa, instrumento validado em adultos jovens brasileiros por Pardini et al. (2001). Considerou-se as atividades realizadas nos domínios lazer e deslocamento nos últimos sete dias em níveis moderados e vigorosos. A determinação do escore semanal de atividade física foi feita por meio da soma do tempo gasto em cada 
atividade. Como convencionado, o tempo das atividades vigorosas é multiplicado por dois (PARDINI et al., 2001). Os entrevistados foram classificados de acordo com o escore obtido em minutos: a) < 150 minutos: nível insuficiente de atividade física; b) $\geq 150$ minutos - ativo fisicamente.

Para avaliar a IC, optou-se por um instrumento que correspondesse aos propósitos da avaliação atitudinal. O instrumento escolhido foi o Body Shape Questionnaire (BSQ), que avalia, por meio 34 questões fechadas, as preocupações com a forma do corpo, a autodepreciação devido à aparência física e à sensação de estar "gordo(a)". Para as respostas, levou-se em consideração a percepção dos estudantes nas quatro últimas semanas. Em cada alternativa escolhida é atribuído um escore que pode variar de 1 a $6(6=$ sempre; $5=$ muito frequentemente; 4 = frequentemente; 3 = às vezes; 2 = raramente; $1=$ nunca). A classificação é realizada mediante a somatória de todos os escores e reflete os níveis de preocupação com a IC. O resultado abaixo de 70 pontos é considerado dentro da normalidade e com ausência de distorção da IC; resultado entre 70 e 90 pontos são classificados como leve distorção da IC; entre 91 e 110 pontos, considera-se distorção moderada; e acima de 110 pontos classifica-se como grave distorção da IC (LAUS; MOREIRA; COSTA, 2009). Esse instrumento foi desenvolvido por Cooper et al. (1987) e validado para uma população de universitários brasileiros por Cordás e Neves (1999), e apresenta medidas psicométricas bastante satisfatórias (COOPER et al. 1987; CORDÁS E NEVES, 1999).

Seguindo os critérios de classificação econômica do Brasil (ABEP, 2010), também foram coletadas informações antropométricas, demográficas e socioeconômicas dos estudantes, como: sexo; idade em anos completos; peso (kg) e estatura (metros); situação conjugal (solteiro, casado, divorciado, viúvo), renda familiar (somatório dos pontos por posse de itens); e grau de instrução do chefe de família (grau incompleto ou completo).

O tratamento estatístico foi realizado por meio do programa Statistical Package for the Social Sciences (SPSS), versão 20. Na primeira etapa foi feita a análise estatística descritiva e inferencial, e os resultados foram expressos em tabelas de frequência. Na segunda fase, utilizou-se o teste de normalidade de Kolmogorov-Smirnov para verificar a normalidade dos dados.

Em seguida, foram realizadas análises de associação entre as variáveis por meio do teste Qui-quadrado para heterogeneidade e tendência linear. Esse teste foi aplicado com o objetivo de verificar a independência entre os resultados do IPAQ e do BSQ com as demais variáveis socioeconômicas e demográficas. Como os dados são lançados em uma tabela de contingência $2 \times 2$, houve a necessidade de transformar as classificações dasvariáveis em escalas binárias.

A última fase das análises estatísticas, a análise multivariada, foi feita por meio da regressão logística binária. Com esse teste foi possível identificar a força e o sentido das interrelações entre variáveis pela Razão de Chances, ou Odds Ratio, com intervalo de confiança de $95 \%$ e um $\mathrm{p} \leq 0,05$. Todas as associações que apresentaram um $\mathrm{p} \leq 0,20$ na análise de associação entre variáveis foram mantidas no modelo de regressão. Entre as variáveis analisadas, a categoria de referência foi aquela que apresentou menor percentual de indivíduos com baixos níveis de atividade física e com distúrbio da IC.

Baseado nas preocupações éticas e metodológicas discutidas nas Diretrizes e Normas Regulamentadoras de Pesquisas envolvendo Seres Humanos (Resolução 466/2012), o presente estudo teve o seu projeto de pesquisa encaminhado à Comissão de Ética em Pesquisa em Seres Humanos da Universidade Estadual Ponta Grossa (UEPG), CAAE: 30336514.0.0000.0105, e foi aprovado sob o parecer $n^{\circ} 625.402$ de 30 de abril de 2014 . 


\section{Resultados}

Dos 498 acadêmicos, 355 concordaram em participar da pesquisa. No entanto, 16 questionários tiveram que ser descartados por não terem sido preenchidos corretamente ou apresentarem questões sem resposta. Assim, os resultados a seguir se referem a 339 acadêmicos de sete cursos de graduação das Faculdades Integradas de Itararé, ano de 2014.

Entre os 339 acadêmicos participantes da pesquisa, 166 (48,9\%) eram homens e 173 $(51,1 \%)$ eram mulheres. A média de idade foi de 22,9 $\pm 5,6$ anos. A média da massa corporal, estatura e IMC autorreferidos foi de $68,2 \pm 14,7 \mathrm{~kg}, 1,69 \pm 0,09 \mathrm{~m}$ e 23,9 $\pm 4,3 \mathrm{~kg} / \mathrm{m}^{2}$, respectivamente. A maioria dos estudantes $(50,1 \%$ da amostra total) apresentou a classificação normal (eutrófico) do estado nutricional avaliado por meio do IMC. Mas 25,1\% e 9,7\% dos jovens apresentaram sobrepeso e obesidade, respectivamente. Entre os estudantes dos cursos de Pedagogia (43,2\%) e Sistemas de Informação (42,8\%) encontrou-se a maior prevalência de indivíduos classificados com sobrepeso e obesidade.

A tabela 1 apresenta os resultados da análise de associação entre o NAF (desfecho) e a percepção da IC, características demográficas (sexo, estado conjugal, IMC e curso) e socioeconômicas (classe econômica).

Tabela 1: Resultados da análise de associação entre o nível de atividade física (NAF) e variáveis independentes. Itararé, São Paulo, Brasil, 2014.

\begin{tabular}{|c|c|c|c|c|c|}
\hline & \multicolumn{2}{|c|}{$\geq 150 \mathrm{~min} / \mathrm{sem}$} & \multicolumn{2}{|c|}{$<150 \mathrm{~min} / \mathrm{sem}$} & \multirow{2}{*}{ p-valor } \\
\hline & $\mathbf{n}$ & $\%$ & $\mathbf{n}$ & $\%$ & \\
\hline \multicolumn{6}{|l|}{ Imagem Corporal (IC) } \\
\hline Sem distúrbio da IC & 190 & 64,2 & 106 & 35,8 & \multirow{2}{*}{0,019} \\
\hline Com distúrbio da IC & 19 & 44,2 & 24 & 55,8 & \\
\hline \multicolumn{6}{|l|}{ Sexo } \\
\hline Masculino & 123 & 74,1 & 43 & 25,9 & \multirow[t]{2}{*}{0,000} \\
\hline Feminino & 86 & 49,7 & 87 & 50,3 & \\
\hline \multicolumn{6}{|l|}{ Estado conjugal } \\
\hline Solteiro & 176 & 64,0 & 99 & 36,0 & \multirow{2}{*}{0,089} \\
\hline Casado & 33 & 51,6 & 31 & 48,4 & \\
\hline \multicolumn{6}{|l|}{ IMC } \\
\hline Abaixo do peso/Normal & 133 & 60,2 & 88 & 39,8 & \multirow{2}{*}{0,519} \\
\hline Acima do peso/Obeso & 76 & 64,4 & 42 & 35,6 & \\
\hline \multicolumn{6}{|l|}{ Classe econômica } \\
\hline A e B & 138 & 63,3 & 80 & 36,7 & \multirow{2}{*}{0,470} \\
\hline C, D e E & 71 & 58,7 & 50 & 41,3 & \\
\hline \multicolumn{6}{|l|}{ Cursos } \\
\hline Administração & 29 & 55,8 & 23 & 44,2 & \multirow{7}{*}{0,015} \\
\hline Ciências Contábeis & 39 & 60,9 & 25 & 39,1 & \\
\hline Direito & 21 & 72,4 & 8 & 27,6 & \\
\hline Educação Física & 54 & 76,1 & 17 & 23,9 & \\
\hline História & 25 & 56,8 & 19 & 43,2 & \\
\hline Pedagogia & 15 & 40,5 & 22 & 59,5 & \\
\hline Sistemas da Informação & 26 & 61,9 & 16 & 38,1 & \\
\hline
\end{tabular}

Nível de significância considerada: $\mathrm{p}<0,05$. 
Pôde-se observar que 209 estudantes $(61,6 \%)$ apresentaram NAF acima do recomendado ( $\geq 150 \mathrm{~min} / \mathrm{sem}$ ). Frente à análise de associação entre as variáveis, verifica-se evidência suficiente para afirmar que o NAF, para essa população específica, apresenta relação de dependência com as variáveis: distúrbio da IC, sexo e o tipo de curso de graduação (Tabela 1).

Tabela 2: Resultados da análise de regressão bruta e ajustada entre nível de atividade física (NAF) insuficiente (< 150 min/sem) e fatores associados. Itararé, São Paulo, Brasil, 2014.

\begin{tabular}{|c|c|c|c|c|c|}
\hline & $\%<150 \mathrm{~min} / \mathrm{sem}$ & $\begin{array}{l}\text { Análise Bruta } \\
\text { RC (IC 95\%) }\end{array}$ & p-valor & $\begin{array}{c}\text { Análise Ajustada } \\
\text { RC (IC 95\%) }\end{array}$ & p-valor \\
\hline \multicolumn{6}{|l|}{ BSQ } \\
\hline Sem distúrbio da IC & 35,8 & 1 & & 1 & \\
\hline Com distúrbio da IC & 55,8 & $2,26(1,18-4,32)$ & 0,013 & $1,56(0,78-3,14)$ & 0,208 \\
\hline \multicolumn{6}{|l|}{ Sexo } \\
\hline Masculino & 25,9 & 1 & & 1 & \\
\hline Feminino & 50,3 & $2,89(1,83-4,57)$ & 0,000 & $2,90(1,71-4,94)$ & 0,000 \\
\hline \multicolumn{6}{|l|}{ Estado conjugal } \\
\hline Solteiro & 36,0 & 1 & & 1 & \\
\hline Casado & 48,4 & $0,60(0,35-1,04)$ & 0,067 & $0,58(0,32-1,05)$ & 0,070 \\
\hline \multicolumn{6}{|l|}{ Cursos } \\
\hline Educação Física & 23,9 & 1 & & 1 & \\
\hline Administração & 44,2 & $2,52(1,16-5,45)$ & 0,019 & $2,42(1,08-5,42)$ & 0,031 \\
\hline Ciências Contábeis & 39,1 & $2,04(0,97-4,27)$ & 0,060 & $1,93(0,89-4,19)$ & 0,094 \\
\hline Direito & 27,6 & $1,21(0,45-3,22)$ & 0,703 & $0,97(0,89-4,19)$ & 0,948 \\
\hline História & 43,2 & $2,41(1,08-5,42)$ & 0,033 & $2,48(1,01-6,13)$ & 0,050 \\
\hline Pedagogia & 59,5 & $4,66(1,98-10,93)$ & 0,000 & $3,06(1,27-7,40)$ & 0,013 \\
\hline Sistemas da Informação & 38,1 & $1,95(0,85-4,47)$ & 0,112 & $2,07(0,89-4,85)$ & 0,092 \\
\hline
\end{tabular}

Nível de significância considerada: $\mathrm{p}<0,05$.

Na segunda etapa dos procedimentos estatísticos para o desfecho atividade física, os resultados da fase ajustada da análise multivariável identificaram que apenas o sexo e o tipo de curso se associaram com o NAF. Como mostrado na tabela 2, o sexo feminino tem chances, aproximadamente, três vezes maior de apresentar níveis insuficientes de prática de atividade física, em relação ao sexo masculino (RC: 2,90; IC95\%: 1,71-4,94). Em relação aos cursos, os estudantes do curso de Administração (RC: 2,42; IC95\%: 1,08-5,42), História (RC: 2,48; IC95\%: 1,01-6,13) e Pedagogia (RC: 3,0; IC95\%: 1,27-7,40) são os que possuem maiores chances de apresentar níveis insuficientes de atividade física quando comparados aos alunos de Educação Física, grupo com menor prevalência de acadêmicos insuficientemente ativos.

Nessa fase da análise, o estado conjugal e a percepção da IC não foram identificados como fatores associados ao NAF, pois a unidade (valor 1) foi incluída no intervalo de confiança dos coeficientes e o nível de significância foi maior que 0,05. Em todos os testes o modelo mostrou-se com boa qualidade de ajuste $(\mathrm{p}<0,01)$.

Em relação à percepção da IC, avaliada por meio do questionário BSQ, pôde-se observar que apenas $43(12,6 \%)$ estudantes foram classificados com presença de distúrbio da IC (leve, moderada ou grave) (Tabela 3). 
Tabela 3: Resultados da análise de dependência entre a percepção da imagem corporal (IC) e variáveis independentes. Itararé, São Paulo, Brasil, 2014.

\begin{tabular}{|c|c|c|c|c|c|}
\hline & \multicolumn{2}{|c|}{ Sem distúrbio da IC } & \multicolumn{2}{|c|}{ Com distúrbio da IC } & \multirow{2}{*}{ p-valor } \\
\hline & $\mathbf{n}$ & $\%$ & $\mathbf{n}$ & $\%$ & \\
\hline \multicolumn{6}{|l|}{ NAF } \\
\hline$\geq 150 \mathrm{~min} / \mathrm{sem}$ & 190 & 90,9 & 19 & 9,1 & \multirow{2}{*}{0,019} \\
\hline$<150 \mathrm{~min} / \mathrm{sem}$ & 106 & 81,5 & 24 & 18,5 & \\
\hline \multicolumn{6}{|l|}{ Sexo } \\
\hline Masculino & 157 & 94,6 & 9 & 5,4 & \multirow{2}{*}{0,000} \\
\hline Feminino & 139 & 80,3 & 34 & 19,7 & \\
\hline \multicolumn{6}{|l|}{ Estado conjugal } \\
\hline Solteiro & 239 & 86,9 & 36 & 13,1 & \multirow{2}{*}{0,797} \\
\hline Casado & 57 & 89,1 & 7 & 10,9 & \\
\hline \multicolumn{6}{|l|}{ IMC } \\
\hline Abaixo do peso/Normal & 203 & 91,9 & 18 & 8,1 & \multirow{2}{*}{0,001} \\
\hline Acima do peso/Obeso & 93 & 78,8 & 25 & 21,2 & \\
\hline \multicolumn{6}{|l|}{ Classe econômica } \\
\hline A e B & 190 & 87,2 & 28 & 12,8 & \multirow{2}{*}{1,000} \\
\hline C, D e E & 106 & 87,6 & 15 & 12,4 & \\
\hline \multicolumn{6}{|l|}{ Cursos } \\
\hline Administração & 44 & 84,6 & 8 & 15,4 & \multirow{7}{*}{0,003} \\
\hline Ciências Contábeis & 55 & 85,9 & 9 & 14,1 & \\
\hline Direito & 27 & 93,1 & 2 & 6,9 & \\
\hline Educação Física & 67 & 94,4 & 4 & 5,6 & \\
\hline História & 36 & 81,8 & 8 & 18,2 & \\
\hline Pedagogia & 26 & 70,3 & 11 & 29,7 & \\
\hline Sistemas da Informação & 41 & 97,6 & 1 & 2,4 & \\
\hline
\end{tabular}

Nível de significância considerada: $\mathrm{p}<0,05$.

Os resultados da análise de dependência apontaram que, a um nível de significância de $95 \%$, o NAF, o sexo, o IMC e o tipo de curso são fatores que influenciaram de maneira significativa na percepção da IC dos estudantes.

A tabela 4 apresenta os resultados do modelo de regressão entre a percepção de distúrbio da IC (desfecho) e os fatores associados no teste de associação. Os dados indicam a presença de um modelo válido para as associações entre o desfecho e o NAF, sexo e IMC ( $\mathrm{p}<$ 0,001). Em contrapartida, o tipo de curso não apresentou associação com o desfecho, uma vez que o teste de Hosmer e Lemeshow indicou a ausência de um modelo válido ( $p>0,01)$. 
Tabela 4: Resultados da análise de regressão bruta e ajustada entre a percepção de distúrbio da IC e fatores associados. Itararé, São Paulo, Brasil, 2014.

\begin{tabular}{lccccc}
\hline & $\begin{array}{c}\text { \% com } \\
\text { distúrbio da } \\
\text { IC }\end{array}$ & $\begin{array}{c}\text { Análise Bruta } \\
\text { RC (IC 95\%) }\end{array}$ & p-valor & $\begin{array}{c}\text { Análise Ajustada } \\
\text { RC (IC 95\%) }\end{array}$ & p-valor \\
\hline NAF & & & & & \\
$\geq 150 \mathrm{~min} / \mathrm{sem}$ & 9,1 & 1 & & 1 & \\
$<150 \mathrm{~min} / \mathrm{sem}$ & 18,5 & $2,26(1,18-4,32)$ & 0,013 & $1,61(0,786-3,301)$ & 0,192 \\
Sexo & & & & & \\
Masculino & 5,4 & 1 & & 1 & \\
Feminino & 19,7 & $4,27(1,98-9,21)$ & 0,000 & $4,05(1,64-10,03)$ & 0,002 \\
IMC & & & & & \\
Abaixo do peso/Normal & 8,1 & 1 & & & \\
Acima do peso/Obeso & 21,2 & $30,3(1,58-5,83)$ & 0,001 & $4,38(2,11-9,10)$ & 0,000 \\
Cursos & & & & & \\
Sistemas da Informação & 2,4 & 1 & & & \\
Administração & 15,4 & $7,45(0,89-62,23)$ & 0,064 & $4,65(0,52-41,34)$ & 0,168 \\
Ciências Contábeis & 14,1 & $6,71(0,82-55,07)$ & 0,076 & $4,07(0,45-36,45)$ & 0,209 \\
Direito & 6,9 & $3,04(0,26-35,16)$ & 0,374 & $1,67(0,131-21,22)$ & 0,693 \\
Educação Física & 5,6 & $2,44(0,26-22,66)$ & 0,430 & $1,75(0,18-17,53)$ & 0,430 \\
História & 18,2 & $9,11(1,09-76,40)$ & 0,042 & $4,61(0,50-42,76)$ & 0,179 \\
Pedagogia & 29,7 & $17,43(2,11-142,40)$ & 0,008 & $5,34(0,562-50,69)$ & 0,145 \\
\hline Nivon & & & & \\
\hline
\end{tabular}

Nível de significância considerada: $\mathrm{p}<0,05$.

Assim, considerando apenas as variáveis que apresentaram associação significativa, é verificado que o sexo feminino, dessa amostra, apresenta quatro vezes mais chances de apresentar algum distúrbio da IC (seja leve, moderada ou grave) em comparação ao sexo masculino (RC: 4,05; IC95\%: 1,64-10,03). Também foi verificado que os indivíduos classificados como acima do peso ou obesos, pelo IMC, apresentam maior chance de insatisfação com o corpo (RC: 4,38; IC95\%: 2,11-9,10) em relação aos que foram classificados como normais ou abaixo do peso.

\section{Discussão}

\section{Relação entre o NAF e fatores associados}

É verificado que entre os acadêmicos com distúrbio da IC há uma prevalência maior de indivíduos com níveis insuficientes de atividade física $(55,8 \%)$. Na análise de associação entre as variáveis, a percepção da IC mostrou-se estar associada com o NAF. Porém, os resultados na fase ajustada da análise de regressão logística binária apontaram que não há evidências suficientes para afirmar, com $95 \%$ de nível de confiança, quantas são as chances de acadêmicos com distúrbio da IC apresentarem níveis insuficientes de atividade física, em comparação ao outro grupo.

Cabe ponderar, no entanto, que a exemplo do que foi encontrado na presente pesquisa, a influência da IC no NAF, em universitários, é observada em outros estudos. De forma linear, os universitários insuficientemente ativos mostram um nível maior de insatisfação com a IC (BRACHT, 2011; HIROTA et al., 2012). Esse fato, tanto no presente 
estudo, como em outros (RECH; ARAÚJO; VANAT, 2010; FERRARI; SILVA; PETROSKI, 2012; HIROTA et al., 2012), é ainda mais representativo entre o sexo feminino.

Em relação ao NAF entre os sexos, é verificado, no presente estudo, maior prevalência do sexo masculino entre os acadêmicos suficientemente ativos $(74,1 \%$ contra 49,7\%). Esses dados confirmam a hipótese de que homens são mais fisicamente ativos (SIMÃO; NAHAS; OLIVEIRA, 2012) e vai ao encontro do verificado em estudos semelhantes (MARCONDELLI; COSTA; SCHIMITZ, 2008; FONTES; VIANNA, 2009).

Também há na produção acadêmica estudo com resultados inversos aos apresentados no presente, como o de Matsudo et al. (2002), no qual foi verificado que 48,7\% das mulheres eram suficientemente ativas, enquanto entre os homens essa taxa foi de $42,5 \%$. Porém, o instrumento utilizado para avaliação do NAF, no referido estudo, foi a versão curta do IPAQ, o qual, segundo Hallal et al. (2010), tende a superestimar o NAF, quando comparado à versão longa. A versão curta contempla todos os domínios do IPAQ (lazer, doméstico, trabalho e deslocamento) em uma mesma questão, e tal fato pode gerar dificuldade por parte do respondente em quantificar, em minutos por semana, as atividades físicas praticadas em todos os domínios de uma única vez (HALLAL et al., 2010).

Ao tentar explicar o fenômeno que faz com que as mulheres apresentem uma prevalência menor de indivíduos suficientemente ativos, Faria e Ramos (2014) advogam que essa diferença entre os sexos se deve a múltiplos fatores; mas o principal deles seria o exercício de dupla jornada de trabalho - trabalho formal e trabalho doméstico - o que contribuiria para a diminuição do tempo livre para que as mesmas pratiquem atividades de lazer, incluindo atividades físicas. Essa afirmação coincide com a de Venturi, Racaman e Oliveira (2004), para os quais, mais da metade das mulheres de todas as regiões do país sacrificam o tempo livre para realizarem atividades domésticas.

No tocante ao NAF entre os cursos de graduação, a presente pesquisa constatou que a maior prevalência de classificados como suficientemente ativos é do curso de Educação Física (76,1\%). Em seguida, com 72,4\%, vieram os alunos de Direito. Resultado semelhante foi observado no estudo de Bielemann et al. (2012), no qual os alunos de Educação Física apareceram como os mais ativos fisicamente em comparação às outras áreas de graduação, com 74,2\%. Os estudos de Silva et al. (2007) e Silva (2012) também registram essa superioridade positiva dos acadêmicos de Educação Física, com 90\% e 89,4\% de fisicamente ativos, respectivamente. Tais resultados, é factível interpretar, encontram justificativa no fato de que os acadêmicos de Educação Física cursam disciplinas práticas durante a graduação; e também tratam de conceitos de educação e promoção da saúde, e da importância do estilo de vida ativo (BIELEMANN et al., 2012; SILVA, 2012).

Em face oposta, o curso de Pedagogia apresentou a maior taxa de indivíduos insuficientemente ativos (40,5\%). Entre os estudantes do curso de Pedagogia, 94,6\% são do sexo feminino e, como apresentado, as mulheres tendem a praticar menos atividade física. Logo, em um curso formado majoritariamente por mulheres, é esperado que, em comparação aos demais, ocorra uma maior taxa de alunos insuficientemente ativos.

\section{Relação entre a IC e fatores associados}

A insatisfação com a IC, denominada de distúrbio da IC, foi de $12,6 \%$ entre os universitários investigados. As análises de associação entre a percepção da IC e as variáveis independentes indicaram que o sexo, o IMC, o NAF e o tipo de curso influenciaram no modo como os estudantes percebem a satisfação com o corpo.

$\mathrm{Na}$ análise multivariável, os resultados revelaram que apenas o sexo e o IMC possuem categorias que apresentam maiores chances de estarem com algum tipo de distúrbio com a IC. Com relação ao sexo, as mulheres possuem quatro vezes mais chances de 
apresentar distúrbio com a IC, em comparação aos homens. Esse achado confirma os resultados descritos em outros estudos (DAMASCENO et al., 2005; RECH; ARAÚJO; VANAT, 2010; SILVA; SAENGER; PEREIRA, 2011).

Em relação à proporção de estudantes do sexo feminino que demonstraram insatisfação com a IC (19,7\%), independentemente de outros fatores sociodemográficos, os resultados do presente estudo não corroboram com a tendência verificada em outros estudos (STIPP; OLIVEIRA, 2003; PONTIERI; LOPES; EÇA, 2007; LAUS; MOREIRA; COSTA, 2009).

Sobre a relação entre a IC e os cursos de graduação, a análise de associação demonstrou que há dependência entre as variáveis $(\mathrm{p}=0,003)$ (Tabela 3), indicando que a distorção da IC existente pode ser dependente do tipo de curso. Tais achados diferem do estudo de Laus, Moreira e Costa (2009), no qual a distorção existente é independente do curso ou da área de estudo. O referido estudo, no entanto, arrolou apenas o sexo feminino, fato esse que pode justificar a diferença (LAUS; MOREIRA; COSTA, 2009).

No presente estudo, na análise estatística mais detalhada, os resultados não foram suficientes para apontar a força da associação e a quantidade de chance que os estudantes dos cursos com maior prevalência de distorção da IC têm de apresentar o desfecho em comparação aos cursos menos prevalentes ( $\mathrm{p}>0,05)$.

No que se refere ao IMC, os resultados mostraram uma prevalência de $50,1 \%$ de indivíduos eutróficos (normais) e 34,8\% com sobrepeso ou obesidade. Tais achados são preocupantes, considerando que outros estudos encontraram porcentagens maiores de universitários classificados dentro do limite de normalidade do IMC (FLORES, 2002; MARCONDELLI; COSTA; SCHMITZ, 2008; MARTINS et al., 2010), com 58\%, 75,8\% $85,9 \%$, respectivamente.

Os resultados da análise ajustada revelam associação significativa, considerando as variáveis IC e IMC. A prevalência de distúrbio da IC foi 4,38 vezes maior nos classificados com sobrepeso e obesidade em comparação aos classificados com IMC normal ou abaixo do peso. Tal associação confirma os levantamentos feitos nos estudos de Silva, Saenger e Pereira (2011) e Rech, Araujo e Vanat (2010), os quais também encontraram associação significativa entre a IC e o estado nutricional de estudantes universitários.

\section{Conclusão}

A realidade empírica para a amostra investigada retrata alta incidência de níveis insuficientes de atividade física $(38,9 \%)$ e baixa prevalência de distorção da IC $(12,6 \%)$, independentemente do sexo, curso de graduação e outros fatores socioeconômicos e demográficos.

As mulheres, no entanto, apresentaram maiores chances tanto para níveis insuficientes de prática de atividade física, quanto de manifestação de insatisfação com a IC, em comparação com aos homens. Dessa forma, é factível a interpretação de que as acadêmicas da amostra pesquisada detêm maior suscetibilidade para o desenvolvimento de comportamentos de risco diretamente ligados ao bem-estar e à qualidade de vida.

O fato de não ter sido realizado o levantamento de informações a respeito dos hábitos alimentares da população em estudo ou feito o controle dessa variável, limitou as conclusões acerca da associação entre o estado nutricional e a percepção da IC. Ainda, considerando a diferença significativa na percepção da IC entre o sexo feminino e o masculino, a avaliação dessa variável por meio de questões que elucidassem se a insatisfação ocorre devido à magreza ou pelo excesso de peso poderia fortalecer ainda mais a discussão sobre a relação entre a IC e o estado nutricional. 


\title{
LEVEL OF PHYSICAL ACTIVITY OF UNIVERSITY STUDENTS WITH AND WITHOUT BODY IMAGE DISORDER
}

\begin{abstract}
The aim of this study was to analyze the relationship between Physical Activity Level (PAL) and the perception of Body Image (BI) in college students. The study included 339 students from seven undergraduate courses, of which 166 were men and 173 were women. The evaluation of the PAL was performed using the IPAQ in its long version. The instrument used to examine the BI was the Body Shape Questionnaire (BSQ). The data reveal a high incidence of insufficient levels of physical activity (38.9\%) and low prevalence of distortion of BI (12.6\%), regardless of gender and undergraduate course. It concludes that the females had higher chances for both insufficient levels of physical activity, as BI disorder.

Keywords: Physical activity; Body image; BMI; University students.
\end{abstract}

\section{NIVEL DE ACTIVIDAD FÍSICA DE ESTUDIANTES UNIVERSITARIOS CON Y SIN DISTURBIO DE LA IMAGEN CORPORAL}

\section{Resumen}

El objetivo del presente estudio fue analizar la relación entre el Nivel de Actividad Física (NAF) y la percepción de la Imagen Corporal (IC) en estudiantes de educación superior. Participaron de este estudio 339 académicos de siete cursos de graduación, de los cuales 166 eran hombres y 173 eran mujeres. La evaluación del NAF fue realizada por medio del IPAQ, en su versión larga. El instrumento utilizado para analizar el IC fue el Body Shape Questionnaire (BSQ). Los dados revelan alta incidencia de niveles insuficientes de actividad física $(38,9 \%)$ y baja prevalencia de distorsión de la IC $(12,6 \%)$, independientemente del género y curso de graduación. Se concluye que el género femenino mostró más chances tanto para niveles insuficientes de práctica de actividad física, cuanto de manifestación de disturbio de la IC.

Palabras clave: Actividad física; Imagen corporal; IMC; Estudiantes universitarios.

\section{Referências}

ASSOCIAÇÃO BRASILEIRA DE EMPRESAS DE PESQUISA. Critério de classificação econômica Brasil: Alterações na aplicação do Critério Brasil, válidas a partir de 01/01/2013, p. 1-15, 2012. Disponível em:<http://www.abep.org/novo/Content.aspx?ContentID=835>. Acesso em: 5 nov. 2013.

ALVARENGA, M. S.; et al. Insatisfação com a Imagem Corporal em Universitárias Brasileiras. Jornal Brasileiro de Psiquiatria, Rio de Janeiro, v. 59, n. 1, p. 44-51, 2010.

BARBOSA, M. R.; MATOS, P. M.; COSTA, M. E. Um olhar sobre o corpo: o corpo ontem e hoje. Psicologia \& Sociedade, v. 23, n. 1, p. 24-34, 2011.

BIELEMANN, R. M.; et al. Prática de atividade física no lazer entre acadêmicos de Educação Física e fatores associados. Revista Brasileira de Atividade Física e Saúde,v. 12, n. 3, p. 65-72, 2012. 
BRACHT, C. M. Percepção da auto-imagem corporal, estado nutricional e prática de atividade física em uma amostra de estudantes da UNIJUÍ. Trabalho de Conclusão de Curso (Graduação). Universidade Regional do Noroeste do Estado do Rio Grande do Sul. Departamento de Ciências da Vida, Curso de Nutrição, Ijuí, 2011.

CAMPANA, A. N. N. B.; TAVARES, M. C. G. C. F. Avaliação da Imagem Corporal: instrumentos e diretrizes para a pesquisa. São Paulo: Phorte, 2009.

COOPER, P. J.; et al. The development and validation of the Body Shape Questionnaire. International Journal of eating disorders, v. 6, n. 4, p. 485-494, 1987.

COQUEIRO, R. S.; et al. Insatisfação com a imagem corporal: avaliação comparativa da associação com estado nutricional em universitários. Revista de Psiquiatria do Rio Grande do Sul, Porto Alegre, v. 30, n.1, p. 31-38, 2008.

CORDÁS, T. A.; NEVES, J. E. P. Escalas de avaliação de transtornos alimentares. Jornal Brasileiro de Psiquiatria, SÃO PAULO, v. 26, n.1, p. 41-47, 1999.

COSTA. K. C. B. C.; et al. Insatisfação Corporal em Estudantes Universitários da Área de Saúde nos Estados de Alagoas e Sergipe. Revista Mudanças - Psicologia da Saúde, São Paulo, v. 18, n. 1-2, p. 1-6, 2010.

DAMASCENO, V.O.; et al. Tipo físico ideal e satisfação com a imagem corporal de praticantes de caminhada. Revista Brasileira de Medicina do Esporte, São Paulo, v.11, n.3, p.181-6, 2005.

FARIA, J. H. de; RAMOS, C. L. Tempo dedicado ao trabalho. Revista Mackenzie, v. 15, n. 4, p. 47-74, 2014.

FERRARI, E. P.; SILVA, D. A. S.; PETROSKI, E. L. Associação entre percepção da imagem corporal e estágios de mudança de comportamento em acadêmicos de educação física. Revista Brasileira de Cineantropometria e Desempenho Humano, v. 14, n. 5, p. 535-544, 2012.

FLORES, A. Prevalência da inatividade física e outros fatores de risco relacionados à saúde na população adulta urbana de Mafra-SC, 2002. [Dissertação de Mestrado] - Universidade Federal de Santa Catarina. Florianópolis: Centro de Desportos, 2002.

FONTES, A. C. D.; VIANNA, R. P. T. Prevalência e fatores associados ao baixo nível de atividade física entre estudantes universitários de uma universidade pública da região Nordeste-Brasil. Revista Brasileira de Epidemiologia. v, 12, p. 20-29, 2009.

GONÇALVES, C. O. Influências da Atividade Física na Imagem Corporal: uma pesquisa bibliográfica. Trabalho de Conclusão de Curso (Graduação) - Faculdade de Educação Física, Universidade Estadual de Campinas. Campinas, São Paulo, 2009.

HALLAL, P. C.; et al. Lecciones aprendidas después de 10 Años del uso de IPAQ en Brasil y Colombia. Journal of Physical Activity \& Health, v. 7, n. 2, p. 259-264, 2010. 
HIROTA, V. B. et al. Nível de Atividade Física e Imagem Corporal de Alunos Ingressos de um Curso de Educação Física da cidade de Santo André - SP. Coleção Pesquisa em Educação Física, São Paulo, v. 11, n. 3, 2012.

LAUS, M. F.; MOREIRA, R. C. M.; COSTA, T. M. B. Diferenças na percepção da imagem corporal, no comportamento alimentar e no estado nutricional de universitárias das áreas de saúde e humanas. Revista de Psiquiatria do Rio Grande do Sul, v. 31, n. 3, p. 192-6, 2009.

MARCONDELLI, P.; COSTA, T. H. M. D.; SCHMITZ, B. D. A. S. Nível de atividade física e hábitos alimentares de universitários do $3^{\circ}$ ao $5^{\circ}$ semestres da área da saúde. Revista Nutrição, v. 21, n. 1, p. 39-47, 2008.

MARTINS, M. C. C.; et al. Pressão arterial, excesso de peso e nível de atividade física em estudantes de universidade pública. Arquivos Brasileiros de Cardiologia, v. 95, n. 2, p. 192$199,2010$.

MARTINS, C. R.; et al. Insatisfação com a Imagem Corporal e Fatores Associados em Universitários. Estudos de Psicologia, v. 17, n. 2, p. 241-246, 2012.

MASTROIANNI, E. C. Q.; et al. A Consciência Corporal na Educação Infantil. In: ZAMBELlO, S.; SAGLIETTI, J. R. (Org.). Núcleos de Ensino artigos 2005. São Paulo: Cultura Acadêmica, 2007, p. 12-30.

MATSUDO, V. K. R.; et al. Nível de atividade física da população do Estado de São Paulo: análise de acordo com o gênero, idade, nível socioeconômico, distribuição geográfica e de conhecimento. Revista Brasileira de Ciência e Movimento, v. 10, p. 41-50, 2002.

MIRANDA, V. P. N. et al. Insatisfação Corporal em Universitários de Diferentes Áreas de Conhecimento. Jornal Brasileiro de Psiquiatria, Rio de Janeiro, v. 61, n. 1, p. 25-32, 2012.

PARDINI, R. et al. Validação do questionário internacional de nível de atividade física (IPAQ-versão 6): estudo piloto em adultos jovens brasileiros. Revista Brasileira de Ciência e Movimento, v. 8, p. 39-44, 2001.

PONTIERI, F. M.; LOPES, P. F.; EÇA, V. B. Avaliação da presença de fatores de para o desenvolvimento de transtornos alimentares em acadêmicos de um curso de Educação Física. Ensaios e Ciência, v. 2, n. 2, p. 29-37, 2007.

QUIOCA, T. et al. Percepção da Imagem e Saúde Corporal dos Universitários do Curso de Educação Física da Unoesc de Joaçaba, SC. Revista Evidência, Joaçaba, v. 10, n. 1-2, p. 43$56,2010$.

RECH, C. R.; ARAÚJO, E. D. S.; VANAT, J. R. Autopercepção da imagem corporal em estudantes do curso de educação física. Revista Brasileira de Educação Física e Esporte, v. 24, n. 2, p. 285-92, 2010.

ROCHA, I. P. Consciência Corporal, Esquema Corporal e Imagem do Corpo. Corpus et Scientia, v. 5, n. 2, p. 26-36, 2009. 
SILVA, M. T. A Relação do Corpo com o Cotidiano da População Adulta e suas Implicações no que se refere ao Contexto Cultural na Contemporaneidade. European Review Of Artistic Studies, Portugal, v. 1, n. 3, p. 94-104, 2010.

SILVA, D. A. S. Nível de atividade física e fatores associados em acadêmicos de educação física de uma universidade pública do nordeste do Brasil. Revista Brasileira de Atividade Física e Saúde, v. 16, n. 3, p. 193-198, 2012.

SILVA, G. S. F. et al. Avaliação do nível de atividade física de estudantes de graduação das áreas saúde/biológica. Revista Brasileira de Medicina do Esporte, v. 13, n. 1, p. 39-42, 2007.

SILVA, T. R.; SAENGER, G.; PEREIRA, E. F. Fatores associados à imagem corporal em estudantes de Educação Física, Motriz, v. 17, n. 4, p. 630-639, out./dez. 2011.

SIMÃO, C. B.; NAHAS, M. V.; OLIVEIRA, E. S. A. D. Atividade física habitual, hábitos alimentares e prevalência de sobrepeso e obesidade em universitários da Universidade do Planalto Catarinense-UNIPLAC, Lages-SC. Revista Brasileira de Atividade Física e Saúde, n. 12, n. 1, p. 3-12, 2012.

STIPP, L. M.; OLIVEIRA, M. R. M. Imagem corporal e atitudes alimentares: diferenças entre estudantes de nutrição e de psicologia. Saúde em Revista, v. 5, n. 9, p. 47-51, 2003.

VENTURI, G.; RACAMÁN, M.; OLIVEIRA, S. A mulher brasileira nos espaços público e privado. São Paulo: Fundação Perseu Abramo, 2004

VIEIRA, V. C. R. et al. Perfil Socioeconômico, Nutricional e de Saúde de Adolescentes recém-ingressos em uma Universidade Pública Brasileira. Revista de Nutrição, CampinasSP, v. 15, n. 3. p. 273-282, 2002.

VIEIRA, F. R. et al. Efeitos das atividades físicas em academias na imagem corporal dos obesos. Fitness \& Performance Journal, n. 1, p. 19-26, 2005.

WILLIAMS, P. A.; CASH, T. F. Effects of a circuit weight training program on the body images of college students. International Journal of Eating Disorders, v. 30, n. 1, p. 75-82, 2001 .

Recebido em: 27/04/2015

Revisado em: 12/10/2015

Aprovado em: 16/10/2015

Endereço para correspondência:

leandro.vargas@uol.com.br

Leandro Martinez Vargas

Faculdades Integradas de Itararé.

João Batista Veiga, 1725

18460-000 - Itararé, SP - Brasil 